\title{
Author Correction: Plant spatial patterns identify alternative ecosystem multifunctionality states in global drylands
}

\author{
Miguel Berdugo, Sonia Kéfi, Santiago Soliveres and Fernando T. Maestre \\ Correction to: Nature Ecology \& Evolution https://doi.org/10.1038/s41559-016-0003, published online 9 January 2017.
}

In the version of this Article originally published, the values of two of the functions used to calculate the multifunctionality index were incorrect. In particular, the values of phosphatase activity and inorganic phosphorus were a copy of total phosphorus and beta glucosidade activity, respectively, in the database due to translocation of columns in an intermediate step for the calculation of multifunctionality. This mistake results in the overall values of the multifunctionality index (which is calculated as the average of $Z$-score values of all functions) to be slightly different from those reported in the paper. The main changes resulting from using the correct dataset are: (i) there is less evidence for two multifunctionality states than previously shown, although this variable is still clearly bimodal, (ii) the two multifunctionality states co-occur over a shorter gradient of aridity values $(0.75-0.80)$ than the ones shown in the original manuscript $(0.60-0.80)$ and (iii) some of the relationships between functions shown in Fig. 3 have changed and now in the low-multifunctionality states there are more connections between functions. Despite these changes, the main results and conclusions from the original paper remained consistent after correcting our mistake. As stated and discussed in the original manuscript, there are two multifunctionality states in global drylands, the co-occurrence of which is preceded by an abrupt decrease of the power law range (PLR). Plant cover is the best predictor for linear changes in multifunctionality but only spatial patterns can detect shifts between these two multifunctionality states, which highlights the potential use of spatial patterns as early warning signals. Despite the changes observed in the relationships between some of the functions studied, the functions related to the $\mathrm{C}$ and $\mathrm{N}$ cycles exhibited fewer connections in high- than in lowmultifunctionality states (as was originally discussed in the paper).

The corrections made in the database affect Figs 3 and 4 of the manuscript, as well as Supplementary Figs 3, 4, 5, 6 and 9. With the results of the corrected dataset, the next sentences introducing some results of the manuscript changed slightly and should be read as follows:

(1) In the sentence "This analysis revealed a bimodal distribution of multifunctionality values in our sites (the Akaike information criterion (AIC) and Bayesian information criterion (BIC) values with one mode (respectively 220.8 and 226.3) were greater than with two modes (respectively 199.8 and 213.6, Fig. 3a), which can be interpreted as the existence of two multifunctionality alternative states in global drylands." the values should have read "one mode (respectively 173.5 and 179.3" and "two modes, (respectively 165.6 and 178.9, Fig. 3a)".

(2) "the PLR showed an abrupt decrease that matched the co-occurrence of the two multifunctionality states observed (Fig. 4)" should be: "the PLR showed an abrupt decrease that preceded the co-occurrence of the two multifunctionality states observed (Fig. 4)".

(3) "Indeed, the type of patch size distribution was significantly associated with the two multifunctionality states observed $\left(\chi^{2}=6.64\right.$, $P=0.01$ ) and this association was even stronger at aridity levels at which the two multifunctionality states co-occurred (Supplementary Fig. 9)", should be: "Indeed, the type of patch size distribution was marginally associated with the two multifunctionality states observed $\left(\chi^{2}=3.70, P=0.05\right)$ and this association was significant at aridity levels preceding the two multifunctionality states (Supplementary Fig. 9)".

(4) "In the high multifunctionality sites, these functions were strongly correlated and multifunctionality was co-driven by many of them (Fig. 3b). In the low multifunctionality sites, organic $\mathrm{C}$ and total $\mathrm{N}$ contents were the main drivers of multifunctionality and functions were loosely linked to each other (Fig. 3b)", should be: "In the high multifunctionality sites, these functions were strongly correlated and phosphorus functions were loosely linked to other functions (Fig. 3b). In the low multifunctionality sites, organic $\mathrm{C}$ and total $\mathrm{N}$ contents were the main drivers of multifunctionality and phosphorus functions were strongly linked to other functions (Fig. 3b)".

(5) In the sentence "They suggest that ecosystems with aridity levels between 0.6 and 0.8 (in the transition zone between semi-arid and arid drylands) may undergo different multifunctionality states, with large contrasts in soil fertility, nutrient capture and nutrient cycling", the aridity levels should have read "between 0.75 and 0.8 ".

We include below the corrected versions of the affected Figs 3 and 4, which have also been corrected in the original article. The corrected Supplementary Figs 3, 4, 5, 6 and 9 are available as a supplementary document to this correction notice, and have been corrected in the Article's Supplementary Information file. Note also that Supplementary Table 1 has been changed to report the correct values of phosphatase activity (PA) and inorganic phosphorus (IP). In the original version, these values were mistakenly duplicated with the values of total phosphorus (TP) and beta glucosidades activity (BGA), respectively. The link to the figshare dataset with the corrected data may be found at https://doi.org/10.6084/m9.figshare.5086540. We apologize for any confusion this may have caused.

Supplementary information is available for this paper at https://doi.org/10.1038/s41559-017-0382-5. 
a

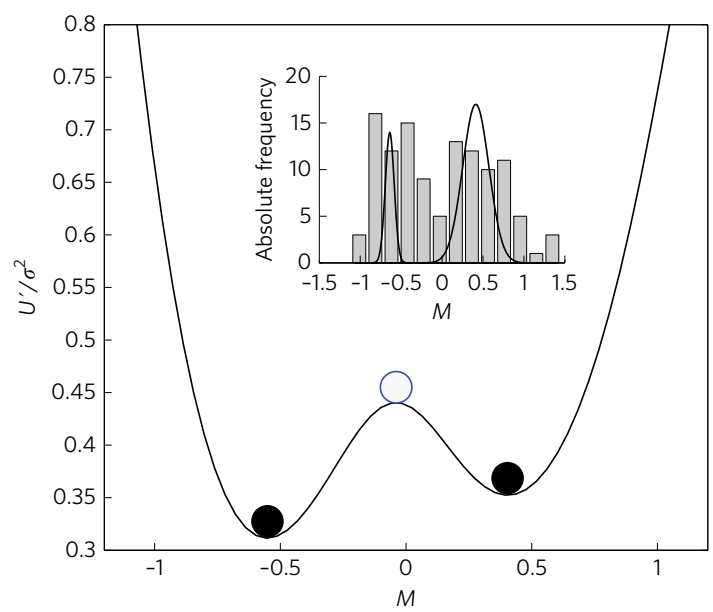

b

b multifunctionality

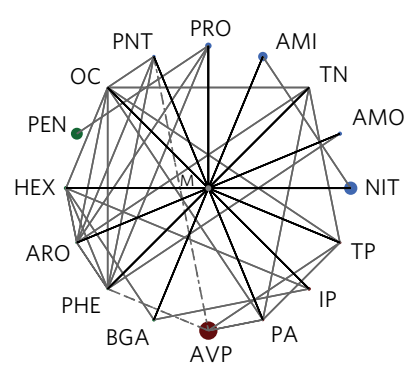

Original Figure 3

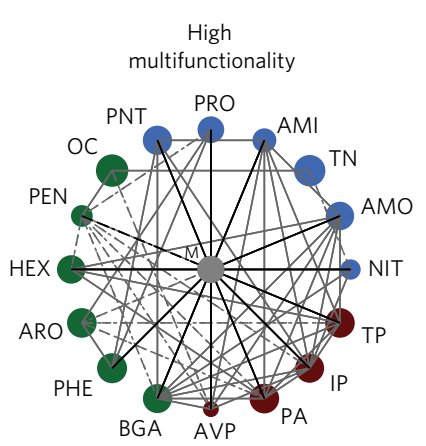

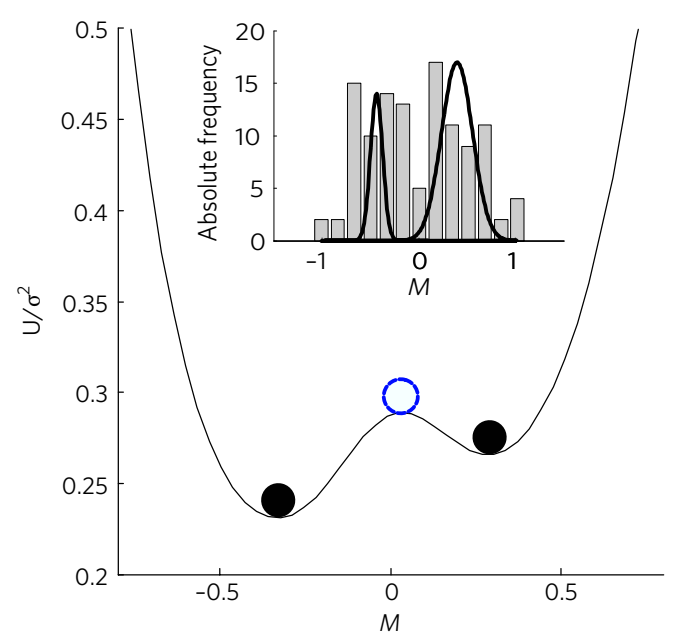
b functionality

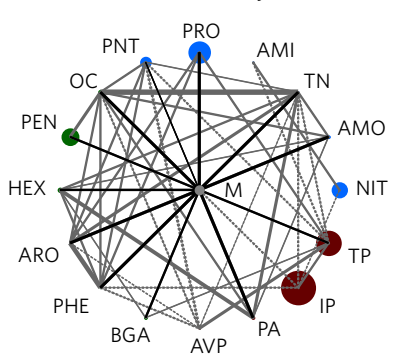

High functionality

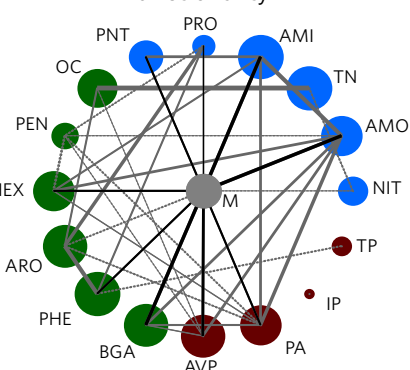

Fig. 3 | Original and corrected. 
Original Figure 4
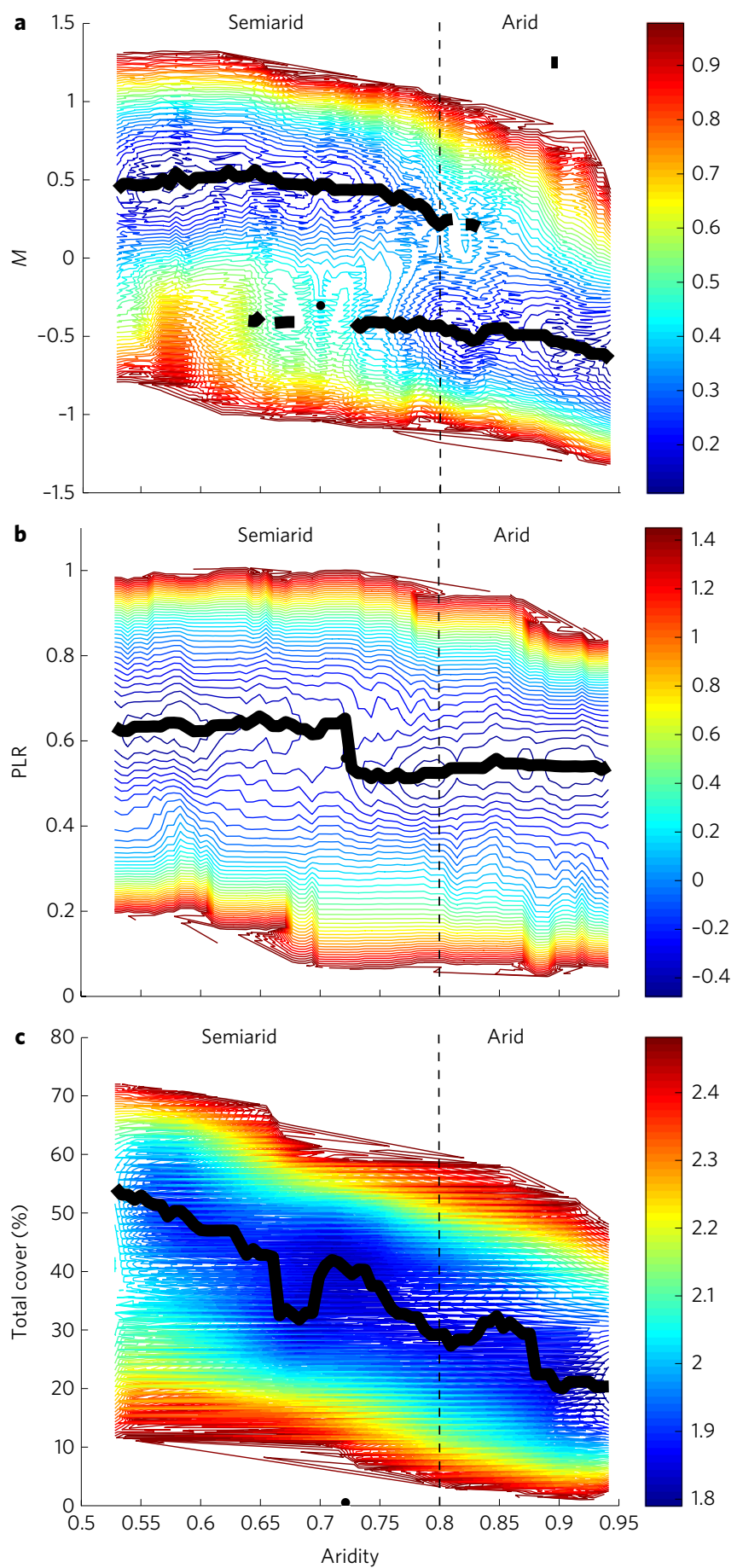

Corrected Figure 4

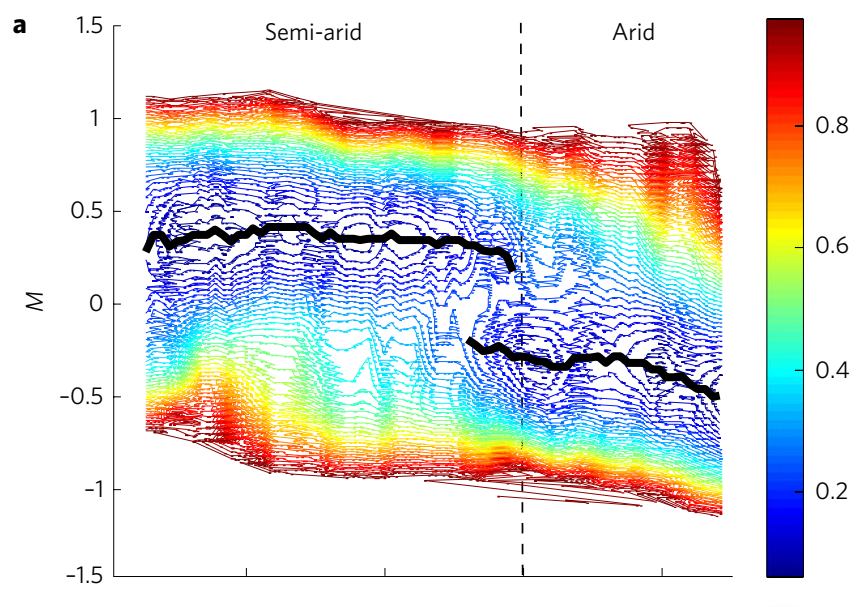

b
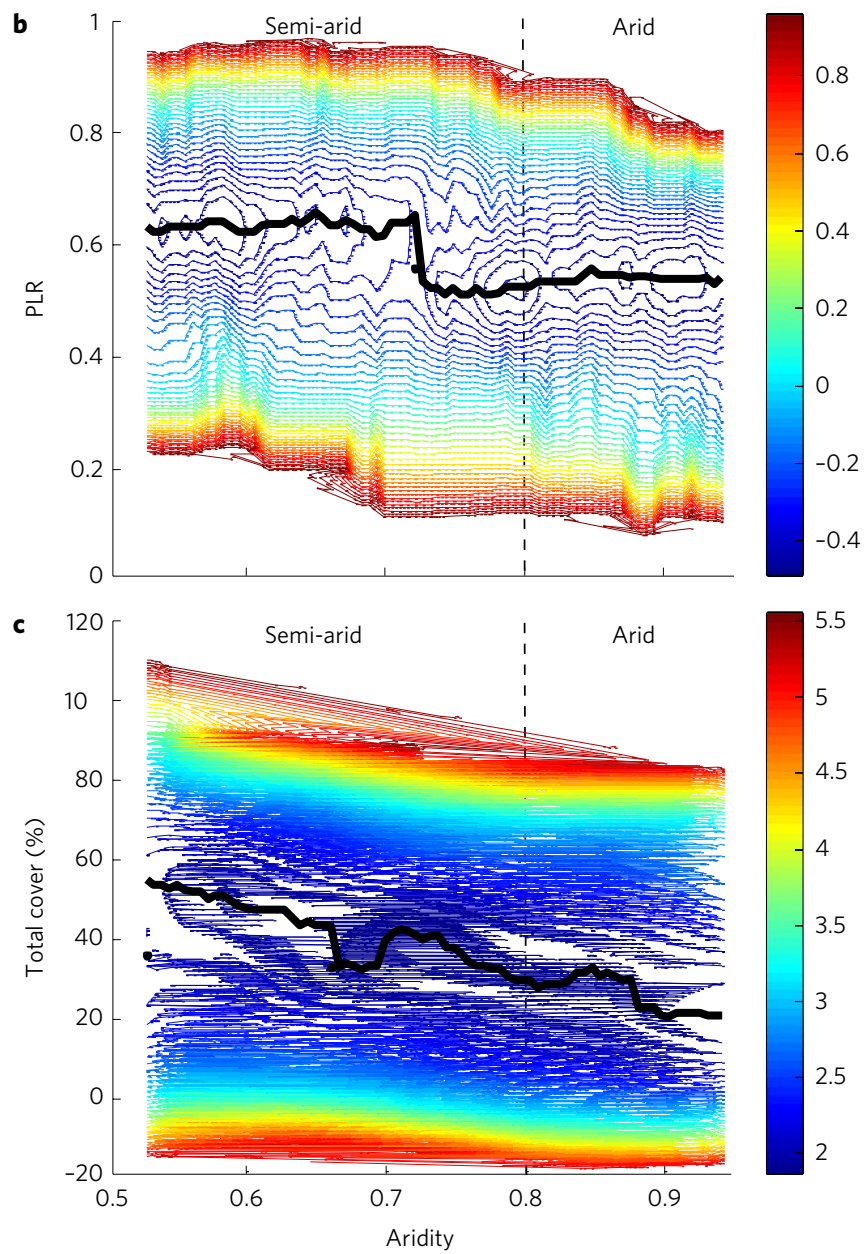

Fig. 4 | Original and corrected. 